ISSN 2078-3744. Вісник Львів. ун-ту. Серія мех.-мат. 2018. Випуск 86. С. 51-62

Visnyk of the Lviv Univ. Series Mech. Math. 2018. Issue 86. P. 51-62

http://publications.lnu.edu.ua/bulletins/index.php/mmf

doi: http://dx.doi.org/10.30970/vmm.2018.86.051-062

УДК $512+514+515.1$

\title{
BORNOLOGICAL, COARSE AND UNIFORM GROUPS
}

\author{
To Taras Banakh and Michael Zarichnyi on their birthdays
}

\section{Igor PROTASOV}

Taras Shevchenko National University of Kyiv, Academic Glushkov Pr. 4d, 03680 Kyiv, Ukraine e-mail: i.v.protasov@gmail.com

We survey and analyze different ways in which bornologies, coarse structures and uniformities on a group agree with the group operations.

Key words: bornology, coarse structure, uniformity, Stone-Čech compactification.

\section{BORNOLOGICAL GROUPS}

1.1. Bornological spaces. A family $\mathcal{I}$ of subsets of a set $X$ is called an ideal (in the Boolean algebra $\mathcal{P}_{X}$ of all subsets of $X$ ) if $\mathcal{I}$ is closed under formation of finite unions and subsets. If $\bigcup \mathcal{I}=X$ then $\mathcal{I}$ is called a bornology, so a bornology is an ideal containing the ideal $[X]^{<\omega}$ of all finite subsets of $X$.

A bornological space is a pair $(X, \mathcal{B})$ consisting of a set $X$ and a bornology $\mathcal{B}$ on $X$. Any set $Y \in \mathcal{B}$ is called bounded. If $X \in \mathcal{B}$, then the bornological space $(X, \mathcal{B})$ is bounded.

Any subset $Y \subset X$ of a bornological space $(X, \mathcal{B})$ carries the subbornology

$$
\mathcal{B} \mid Y:=\{B \in \mathcal{B}: B \subset Y\},
$$

induced by $\mathcal{B}$.

A bornology $\mathcal{B}$ on $X$ is called

- tall if $\mathcal{B} \mid Y \neq[Y]^{<\omega}$ for any infinite subset $Y$;

- antitall if any subset $Y \notin \mathcal{B}$ of $X$ contains an infinite subset $Z \subseteq Y$ such that $\mathcal{B} \mid Z=[Z]^{<\omega}$. 
By [15, Proposition 1], every bornology is the meet of some tall and antitall bornologies.

A family $\mathcal{B}^{\prime} \subseteq \mathcal{B}$ is called a base of a bornology $\mathcal{B}$ if each set $B \in \mathcal{B}$ is contained in some set $B^{\prime} \in \mathcal{B}^{\prime}$. Every bornology with a countable base is antitall. In particular, the bornology of all bounded subsets of a metric space is antitall. We note also that for every bornology $\mathcal{B}$ on $X$ with a countable base, there exists a metric $d$ on $X$ such that $\mathcal{B}$ is a bornology of all bounded subsets of $(X, d)$.

The product of a family of bornological spaces $\left(X_{\alpha}, \mathcal{B}_{\alpha}\right), \alpha \in A$, is the Cartesian product $\prod_{\alpha \in A} X_{\alpha}$ of their supports endowed with the bornology generated by the base $\left\{\prod_{\alpha \in A} B_{\alpha}:\left(B_{\alpha}\right)_{\alpha \in A} \in \prod_{\alpha \in A} \mathcal{B}_{\alpha}\right\}$.

A mapping $f: X \rightarrow Y$ between two bornological spaces $\left(X, \mathcal{B}_{X}\right)$ and $\left(Y, \mathcal{B}_{Y}\right)$ is called bornologous if $\left\{f(B): B \in \mathcal{B}_{X}\right\} \subset \mathcal{B}_{Y}$.

A variety is a class of bornological spaces closed under formation of subspaces, products and bornologous images.

We denote by $\mathfrak{M}_{\text {single }}$ the variety of all singletons, $\mathfrak{M}_{\text {bound }}$ the variety of all bounded bornological spaces, $\mathfrak{M}_{\kappa}$ the variety of all $\kappa$-bounded spaces. For an infinite cardinal $\kappa$, a bornological space $(X, \mathcal{B})$ is called $\kappa$-bounded if each subset $B \subset X$ of cardinality $|B|<\kappa$ is bounded.

Every variety of bornological spaces coincides with one of the varieties in the chain:

$$
\mathfrak{M}_{\text {single }} \subset \mathfrak{M}_{\text {bound }} \subset \cdots \subset \mathfrak{M}_{\kappa} \subset \cdots \subset \mathfrak{M}_{\omega}
$$

see the proof of Theorem 2 in [16].

1.2. Bornologies on groups. A bornology on a group $G$ is called right (left) invariant if $\mathcal{B} g \subseteq \mathcal{B}$ (resp. $g \mathcal{B} \subseteq \mathcal{B}$ ) for each every $g \in G$. Here $\mathcal{B} g=\{B g: B \in \mathcal{B}\}$ and $g \mathcal{B}=\{g B: B \in \mathcal{B}\}$. A group $G$ endowed with a right (left) invariant bornology is called a right (left) bornological group.

We say that a group $G$ endowed with a bornology $\mathcal{B}$ is a bornological group if the group multiplication and inversion are bornologous mapping. In this case, $\mathcal{B}$ is called a group bornology. We note that $\mathcal{B}$ is a group bornology if and only if for any $A, B \in \mathcal{B}$ we have $A B^{-1} \in \mathcal{B}$.

1.3. Duality. Now we endow every group $G$ with the discrete topology and identify the Stone-Cech compactification $\beta G$ of $G$ with the set of all ultrafilters on $G$. Then the family $\{\bar{A}: A \subseteq G\}$, where $\bar{A}=\{p \in \beta G: A \in p\}$, forms a base of the topology of $\beta G$. Given a filter $\varphi$ on $G$, we denote $\bar{\varphi}=\cap\{\bar{A}: A \in \varphi\}$, so $\varphi$ defines the closed subset $\bar{\varphi}$ of $\beta G$, and each closed subset $K$ of $\beta G$ can be obtained in this way: $K=\bar{\varphi}$, where $\bar{\varphi}=\{A \subseteq G: K \subseteq \bar{A}\}$.

We use the standard extension [8, Section 4.1] of the multiplication on $G$ to the semigroup multiplication on $\beta G$ such that for every $p \in \beta G$ the right shift $\beta G \rightarrow \beta G$, $x \mapsto x p$, is continuous, and for every $g \in G$ the left shift $\beta G \rightarrow \beta G, x \mapsto g x$, is continuous. For ultrafilters $p, q \in \beta G$ their product $p q$ in $\beta G$ is defined by the formula

$$
p q=\left\{\bigcup_{x \in P} x Q_{x}: P \in p,\left\{Q_{x}\right\}_{x \in P} \subset q\right\} .
$$

Let $G^{*}:=\beta G \backslash G$ be the set of all free ultrafilters on $G$. It follows directly from the definition of the multiplication in $\beta G$ that $G^{*}$ and $\overline{G^{*} G^{*}}$ are ideals in the semigroup $\beta G$, and $G^{*}$ is the unique maximal closed ideal in $G$. By Theorem 4.44 from [8], the closure 
$\overline{K(\beta G)}$ of the minimal ideal $K(G)$ of $\beta G$ is an ideal, so $\overline{K(\beta G)}$ is the smallest closed ideal in $\beta G$. For the structure of $\overline{K(\beta G)}$ and some other ideals in $\beta G$ see [8, Sections 4, $6]$.

For an ideal $\mathcal{I}$ on a group $G$ and a closed subset $K$ of $\beta G$, we put

$$
\mathcal{I}^{\wedge}=\{p \in \beta G: \forall A \in \mathcal{I} \quad G \backslash A \in p\} \text { and } K^{\vee}=\{G \backslash A: A \in \varphi, \bar{\varphi}=K\} .
$$

We have the following duality statements:

- $\mathcal{I}$ is left translation invariant if and only if $\mathcal{I}^{\wedge}$ is a left ideal of the semigroup $\beta G$;

- $\mathcal{I}$ is right translation invariant if and only if $\left(\mathcal{I}^{\wedge}\right) G \subseteq \mathcal{I}^{\wedge}$;

- $\left(\mathcal{I}^{\wedge}\right)^{\vee}=\mathcal{I}$

- $\mathcal{I}$ is a bornology if and only if $\mathcal{I}^{\wedge} \subseteq G^{*}$.

Thus, we have the duality between left invariant bornologies on $G$ and closed left ideals of $\beta G$ containing in $G^{*}$. We say that a subset $A$ of a group $G$ is

- large if $G=F A$ for some $F \in[G]^{<\omega}$;

- small if $L \backslash A$ is large for every large subset $L$ of $G$;

- sparse if for every infinite subset $X$ of $G$ there exists a finite subset $F \subset X$ such that $\bigcap_{g \in F} g A$ is finite.

Theorem 1. For every infinite group $G$, the family $\mathcal{S}_{m_{G}}$ of all small subsets of $G$ is a left and right invariant bornology and $\mathcal{S}_{G} \stackrel{\wedge}{K(\beta G)}$.

This is Theorem 4.40 from [8] in the form given in [17, Theorem 12.5].

Theorem 2. For every infinite group $G$, the family $\mathcal{S}_{p_{G}}$ of all sparse subsets of $G$ is a left and right invariant bornology and $\mathcal{S}{ }_{G}^{\wedge}=G^{*} G^{*}$.

This is Theorem 10 from 4 .

More applications of this duality can be found in [18].

Let $\mathcal{B}$ be a group bornology on $G$. By $\left[23, \mathcal{B}^{\wedge}\right.$ is an ideal in $\beta G$ but the converse statement does not hold: $\mathcal{S} m_{G}^{\wedge}$ is an ideal but $\mathcal{S} m_{G}$ is not a group bornology.

1.4. Plenty of bornologies. We say that a left invariant bornology $\mathcal{B}$ of $G$ is maximal if $G \notin \mathcal{B}$ but $G \in \mathcal{B}^{\prime}$ for every left invariant bornology $\mathcal{B}^{\prime}$ on $G$ such that $\mathcal{B} \subsetneq \mathcal{B}^{\prime}$.

Theorem 3. For every infinite group $G$, of cardinality $\kappa$, there are $2^{2^{\kappa}}$ distinct maximal left invariant bornologies on $G$.

Proof. By Theorem 6.30 from [8], there exists a family $\mathfrak{F}$ consisting of $|\mathfrak{F}|=2^{2^{\kappa}}$ pairwise disjoint closed left ideals in $\beta G$. Each $I \in \mathfrak{F}$ contains some minimal closed left ideal $L_{I}$. Then $\left\{L_{I}^{\vee}: I \in \mathfrak{F}\right\}$ is the desired family of maximal left invariant bornologies on $G$.

Theorem 4 ([14]). Every countable group $G$ admits exactly $2^{\mathfrak{c}}$ group bornologies.

By Theorem 6.3.3 from [26], each Abelian group $G$ admits exactly $2^{2^{|G|}}$ group bornologies. However this theorem does not extend to uncountable non-commutative groups. The following (consistent) counterexample was suggested by Taras Banakh.

Example 1. Under $\mathbf{C H}$ there exists a group $G$ of cardinality $|G|=\mathfrak{c}=\omega_{1}$ admitting exactly $2^{\mathfrak{c}}<2^{2^{\mathfrak{c}}}$ group bornologies. 
Proof. In [29] Shelah constructed a CH-example of a group $G$ of cardinality $|G|=\mathfrak{c}=\omega_{1}$ such that $G=A^{6641}$ for any uncountable subset $A \subset G$. This implies that each group bornology $\mathcal{B}$ on $G$ either coincides with $\mathcal{P}_{G}$ or is contained in the bornology $[G] \leq \omega$ of countable subsets of $G$. Thus, the number of group bornologies of Shelah's group $G$ is $\leq 2^{\mid[G]]^{\leq \omega} \mid}=2^{\mathfrak{c}}$. On the other hand, Theorem 4 ensures that this number is $\geq 2^{\mathfrak{c}}$. Therefore, the number of group bornologies of Shelah's group $G$ is equal to $2^{\mathfrak{c}}$, which is strictly smaller than $2^{2^{\mathrm{c}}}$ by known Cantor's Theorem.

Theorem 4 implies that under the assumption $2^{\omega_{1}}=2^{\omega}$, each group $G$ of cardinality $|G|=\omega_{1}$ has exactly $2^{\mathfrak{c}}=2^{2^{|G|}}$ group bornologies. This observation shows that the group from Example 1 cannot be constructed in ZFC.

Theorem 5 ([18]). For every infinite group $G$, the following statements hold:

(i) if $\mathcal{B}$ is a left invariant bornology on $G$ and $\mathcal{B} \neq[G]^{<\omega}$, then there is a left invariant bornology $\mathcal{B}^{\prime}$ on $G$ such that $[G]^{<\omega} \subsetneq \mathcal{B}^{\prime} \subsetneq \mathcal{B}$;

(ii) if $G$ is either countable or Abelian and $\mathcal{B}$ is a left and right invariant bornology on $G$ such that $\mathcal{B} \neq[G]<\omega$ then there is a left and right invariant bornology $\mathcal{B}^{\prime}$ on $G$ such that $[G]^{<\omega} \subsetneq \mathcal{B}^{\prime} \subsetneq \mathcal{B}$;

(iii) if $G$ is the group $S_{\kappa}$ of all permutations of an infinite cardinal $\kappa$ then there exists a left and right invariant bornology $\mathcal{B}$ on $G$ such that $[G]^{<\omega} \subsetneq \mathcal{B}$ and there are no left and right invariant bornologies on $G$ between $[G]^{<\omega}$ and $\mathcal{B}$.

Theorem 6. Let $G$ be an infinite group and let $\mathcal{B}$ be a group bornology of $G$ such that $\mathcal{B} \neq[G]^{<\omega}$. If $G$ is either countable or Abelian then there is a group bornology $\mathcal{B}^{\prime}$ such that $[G]^{<\omega} \subsetneq \mathcal{B}^{\prime} \subsetneq \mathcal{B}$.

This is Theorem 6.4.1. from [26]. We do not know (Question 6.4.1. in [26]) if Theorem 6 remains true for every infinite group $G$, in particular, for $G=S_{\kappa}$.

By [17, Theorem 12.9], every infinite group can be partitioned into countably many small subsets.

Question 1. Given an infinite group $G$, do there exist a small subset $S$ and a countable subset $A$ of $G$ such that $\{a S: a \in A\}$ is a covering (partition) of $G$ ?

This is so if either $G$ is amenable or $G$ has a subgroup of countable index.

\section{CoArse groups}

2.1. Balleans and coarse spaces. For a set $X$ a subset $\varepsilon \subset X \times X$ containing the diagonal $\triangle_{X}:=\{(x, x): x \in X\}$ is called an entourage on $X$. For two entourages $\varepsilon, \varepsilon^{\prime}$ on $X$ the sets

$$
\varepsilon \circ \varepsilon^{\prime}=\left\{(x, z): \exists y \in X(x, y) \in \varepsilon,(y, z) \in \varepsilon^{\prime}\right\} \quad \text { and } \quad \varepsilon^{-1}=\{(y, x):(x, y) \in \varepsilon\} .
$$

also are entourages on $X$.

A family $\mathcal{E}$ of entourages on $X$ is called a ball structure if it satisfies two axioms:

(a) for any $\varepsilon, \delta \in \mathcal{E}$ the entourage $\varepsilon \circ \delta^{-1}$ is contained in some $\lambda \in \mathcal{E}$;

(b) $\bigcup \mathcal{E}=X \times X$.

A ball structure $\mathcal{E}$ on $X$ is called a coarse structure if it satisfies one additional axiom: 
(c) if $\varepsilon \in \mathcal{E}$ and $\Delta_{X} \subseteq \delta \subseteq \varepsilon$, then $\delta \in \mathcal{E}$.

It follows that each coarse structure is a ball structure and each ball structure $\mathcal{E}$ on $X$ is a base of the unique coarse structure

$$
\downarrow \mathcal{E}=\left\{\delta: \exists \varepsilon \in \mathcal{E} \quad \Delta_{X} \subseteq \delta \subseteq \varepsilon\right\} .
$$

A subfamily $\mathcal{E}^{\prime} \subseteq \mathcal{E}$ is called a base of a coarse structure $\mathcal{E}$ if for every $\varepsilon \in \mathcal{E}$ is contained in some $\delta \in \mathcal{E}^{\prime}$.

A ballean is a pair $(X, \mathcal{E})$ consisting of a set $X$ and a ball structure $\mathcal{E}$ on $X$. If the ball structure $\mathcal{E}$ is a coarse structure, then the ballean $(X, \mathcal{E})$ is called a coarse space. We note that coarse spaces can be considered as asymptotic counterparts of uniform spaces and balleans can be defined in terms of balls, see [17], 26].

Let $\mathcal{E}$ be a ball structure on a set $X$. For every $x \in X$ and $\varepsilon \in \mathcal{E}$ the set $B(x, \varepsilon):=$ $\{y \in X:(x, y) \in \varepsilon\}$ is called the ball of radius $\varepsilon$ centered at $x$.

A subset $Y$ of $X$ is called bounded if there exist $x \in X$ and $\varepsilon \in \mathcal{E}$ such that $Y \subseteq B(x, \varepsilon)$. The coarse structure $\mathcal{E}=\left\{\varepsilon \in X \times X: \triangle_{X} \subseteq \varepsilon\right\}$ is the unique coarse structure such that $(X, \mathcal{E})$ bounded.

For a ballean $(X, \mathcal{E})$, each subset $Y \subseteq X$ carries the induced ball structure $\mathcal{E}\lceil Y:=$ $\{\varepsilon \cap(Y \times Y): \varepsilon \in \mathcal{E}\}$. The ballen $(Y, \mathcal{E}\lceil Y)$ is called a subballean of $(X, \mathcal{E})$.

A subset $Y$ of a ballean $X$ is called large (or coarsely dense) if there exists $\varepsilon \in \mathcal{E}$ such that $X=B(Y, \varepsilon)$ where $B(Y, \varepsilon)=\bigcup_{y \in Y} B(y, \varepsilon)$.

Let $(X, \mathcal{E}),\left(X^{\prime}, \mathcal{E}^{\prime}\right)$ be balleans. A mapping $f: X \rightarrow X^{\prime}$ is called coarse if for every $\varepsilon \in \mathcal{E}$ there exists $\varepsilon^{\prime} \in \mathcal{E}^{\prime}$ such that, for every $x \in X$, we have $f(B(x, \varepsilon)) \subseteq B\left(f(x), \varepsilon^{\prime}\right)$. If $f$ is surjective and coarse, then $\left(X^{\prime}, \mathcal{E}^{\prime}\right)$ is called a coarse image of $(X, \mathcal{E})$. If $f$ is a bijection such that $f$ and $f^{-1}$ are coarse mappings, then $f$ is called an asymorphism. Two balleans $(X, \mathcal{E}),\left(X^{\prime}, \mathcal{E}^{\prime}\right)$ are called coarsely equivalent if there exist large subsets $Y \subseteq X$, $Y^{\prime} \subseteq X^{\prime}$ such that the balleans $\left(Y, \mathcal{E}\lceil Y)\right.$ and $\left(Y^{\prime}, \mathcal{E}^{\prime} \uparrow Y^{\prime}\right)$ are asymorphic.

To conclude the coarse vocabulary, we take a family $\left\{\left(X_{\alpha}, \mathcal{E}_{\alpha}\right): \alpha<\kappa\right\}$ of balleans and define their product $\prod_{\alpha<\kappa}\left(X_{\alpha}, \mathcal{E}_{\alpha}\right)$ as the Cartesian product $\prod_{\alpha<\kappa} X_{\alpha}$ endowed with the ball structure consisting of the entourages

$$
\left\{\left(\left(x_{\alpha}\right),\left(y_{\alpha}\right)\right): \forall \alpha \in A\left(x_{\alpha}, y_{\alpha}\right) \in \varepsilon_{\alpha}\right\}
$$

where $\left(\varepsilon_{\alpha}\right)_{\alpha \in A} \in \prod_{\alpha \in A} \mathcal{E}_{\alpha}$.

For lattices of coarse structures and varieties of coarse spaces, see [19] and [16].

For every ballean $(X, \mathcal{E})$, the family of all bounded subsets of $X$ is a bornology. On the other hand, for every bornology $\mathcal{B}$ on $X$, there is the smallest by inclusion coarse structure $\mathcal{E}_{\mathcal{B}}$ on $X$ such that $\mathcal{B}$ is the bornology of all bounded subsets of $\left(X, \mathcal{E}_{\mathcal{B}}\right)$. A coarse structure $\mathcal{E}$ on $X$ is of the form $\mathcal{E}_{\mathcal{B}}$ if and only if $(X, \mathcal{E})$ is thin: for every $\varepsilon \in \mathcal{E}$, there exists a bounded subset $A$ of $(X, \mathcal{E})$ such that $B(x, \varepsilon)=\{x\}$ for all $x \in X \backslash A$. The thin coarse structures are also called discrete.

2.2. Coarse structures on groups. We remind that a bornology $\mathcal{I}$ on a group $G$ is a group bornology if and $A B^{-1} \in \mathcal{I}$ for all $A, B \in \mathcal{I}$. A group bornology $\mathcal{I}$ is called invariant if $\bigcup_{g \in G} g^{-1} A g \in \mathcal{I}$ for each $A \in \mathcal{I}$.

Let $X$ be a $G$-space with an action $G \times X \rightarrow X,(g, x) \mapsto g x$, of a group $G$. We assume that $G$ acts on $X$ transitively, take a group bornology $\mathcal{I}$ on $G$ and consider the 
coarse structure $\mathcal{E}(G, \mathcal{I}, X)$ on $X$ generated by the base consisting of the entourages $\varepsilon_{A}:=\{(x, y) \in X \times X: y \in\{x\} \cup A x\}$. In this case $B\left(x, \varepsilon_{A}\right)=\{x\} \cup A x$.

By [10, Theorem 1], for every coarse structure $\mathcal{E}$ on $X$, there exist a group $G$ of permutations of $X$ and a group ideal $\mathcal{I}$ on $G$ such that $\mathcal{E}=\mathcal{E}(G, \mathcal{I}, X)$.

Now let $X=G$ and $G$ acts on $X$ by the left shifts. We denote $\mathcal{E}_{\mathcal{I}}=\mathcal{E}(G, \mathcal{I}, G)$. Thus, every group bornology $\mathcal{I}$ on $G$ turns $G$ into the coarse space $\left(G, \mathcal{E}_{\mathcal{I}}\right)$. We note that a subset $A$ of $G$ is bounded in $\left(G, \mathcal{E}_{\mathcal{I}}\right)$ if and only if $A \in \mathcal{I}$.

A group $G$ endowed with a coarse structure $\mathcal{E}$ is called left (right) coarse group if, for every $\varepsilon \in \mathcal{E}$, there exists $\varepsilon^{\prime} \in \mathcal{E}$ such that $g B(x, \varepsilon) \subseteq B\left(g x, \varepsilon^{\prime}\right)$ (resp. $B(x, \varepsilon) g \subseteq$ $B\left(x g, \varepsilon^{\prime}\right)$ ) for all $x, g \in G$. Equivalently, $(G, \mathcal{E})$ is a left (right) coarse group if $\mathcal{E}$ has a base consisting of left (right) invariant entourages. An entourage $\varepsilon$ is left (right) invariant if $g \varepsilon=\varepsilon$ (resp. $\varepsilon g=g$ ) for each $g \in G$, where $g \varepsilon=\{(g x, g y):(x, y) \in \varepsilon)\}$ and $\varepsilon g=\{(x g, y g):(x, y) \in \varepsilon)\}$. For finitely generated groups, the right coarse groups $\left(G, \mathcal{E}_{[G]<\omega}\right)$ in metric form play an important role in Geometric Group Theory, see [6. Chapter 4].

A group $G$ endowed with a coarse structure $\mathcal{E}$ is called a coarse group if the group multiplication $(G, \mathcal{E}) \times(G, \mathcal{E}) \rightarrow(G, \mathcal{E}),(x, y) \mapsto x y$, and the inversion $(G, \mathcal{E}) \rightarrow(G, \mathcal{E})$, $x \mapsto x^{-1}$, are coarse mappings. In this case, $\mathcal{E}$ is called a group coarse structure.

The following two statements are taken from [22, see also [26, Chapter 6].

Proposition 1. A group $G$ endowed with a coarse structure $\mathcal{E}$ is a right coarse group if and only if there exists a group bornology $\mathcal{I}$ on $G$ such that $\mathcal{E}=\mathcal{E}_{\mathcal{I}}$.

Proposition 2. For a group $G$ endowed with a coarse structure $\mathcal{E}$, the following conditions are equivalent:

(i) $(G, \mathcal{E})$ is a coarse group;

(ii) $(G, \mathcal{E})$ is a left and right coarse group;

(iii) there exists an invariant group bornology $\mathcal{I}$ on $G$ such that $\mathcal{E}=\mathcal{E}_{\mathcal{I}}$.

Applying Theorem 1.4, we get $2^{2^{\aleph_{0}}}$ distinct right coarse structures on any countable group. For every infinite group $G$ and any infinite cardinal $\kappa \leqslant|G|$, the bornology $[G]^{<\kappa}$ defines an unbounded right coarse structure on $G$. But if $G$ has only two conjugated classes then there is only one, bounded, group coarse structure on $G$.

2.3. Asymorphisms. For an infinite cardinal $\kappa$, we say that two groups $G$ and $H$ are $\kappa$ asymorphic (resp. $\kappa$-coarsely equivalent) if the right coarse structures on $G$ and $H$ defined by the bornologies $[G]^{<\kappa}$ and $[H]^{<\kappa}$ are asymorphic (resp. coarsely equivalent). In the case $\kappa=\aleph_{0}, G$ and $H$ are called finitarily asymorphic and finitarily coarsely equivalent, respectively.

Let us recall that a group $G$ is locally finite if each finite subset of $G$ is contained in a finite subgroup of $G$. A classification of countable locally finite groups up to finitary asymorphisms is obtained in [12] (cf. [17, p. 103]).

Theorem 7. Two countable locally finite groups $G_{1}$ and $G_{2}$ are finitarily asymorphic if and only if the following conditions hold:

(i) for every finite subgroup $F_{1} \subset G_{1}$, there exists a finite subgroup $F_{2}$ of $G_{2}$ such that $\left|F_{1}\right|$ is a divisor of $\left|F_{2}\right|$; 
(ii) for every finite subgroup $F_{2}$ of $G_{2}$, there exists a finite subgroup $F_{1}$ of $G_{1}$ such that $\left|F_{2}\right|$ is a divisor of $\left|F_{1}\right|$.

It follows that there are continuum many distinct types of countable locally finite groups and each group is finitarily asymorphic to some direct sum of finite cyclic groups.

The following coarse classification of countable Abelian groups is obtained in [2].

Theorem 8. Two countable groups $G$ and $H$ are finitarily coarsely equivalent if and only if the torsion-free ranks of $G$ and $H$ coincide and $G, H$ are both either finitely generated or infinitely generated. valent.

In particular, any two countable torsion Abelian groups are finitarily coarsely equi-

For $\kappa$-asymorphisms, we have the following two results.

Theorem 9 (25]). For any uncountable cardinal $\kappa$, any two groups $G, H$ of cardinality $|G|=\kappa=|H|$ are $\kappa$-asymorphic.

Theorem 10 ([24]). Let $\kappa$ be a cardinal and $G$ be an Abelian group of cardinality $|G| \geq \kappa$. The group $G$ is $\kappa$-asymorphic to a free Abelian group. If $\kappa<|G|$ or $\kappa=|G|$ is a singular cardinal, then $G$ is not not $\kappa$-coarsely equivalent to a free group. In particular, $G$ is not $\kappa$-asymorphic to a free group.

Theorem $11([20])$. For every countable group $G$ there are continuum many distinct classes of finitarily coarsely equivalent subsets of $G$.

2.4. Free coarse groups. A class $\mathfrak{M}$ of groups is a variety if $\mathfrak{M}$ is closed under subgroups, products and homomorphic images. We assume that $\mathfrak{M}$ is non-trivial (i.e. there exists $G \in \mathfrak{M}$ such that $|G|>1$ ) and recall that the a group $F_{\mathfrak{M}}(X)$ in the variety $\mathfrak{M}$ is defined by the following conditions: $F_{\mathfrak{M}}(X) \in \mathfrak{M}, X \subset F_{\mathfrak{M}}(X), X$ generates $F_{\mathfrak{M}}(X)$ and every mapping $X \rightarrow G, G \in \mathfrak{M}$ can be extended to homomorphism $F_{\mathfrak{M}}(X) \rightarrow G$.

For a coarse space $(X, \mathcal{E})$, a free coarse group $F_{\mathfrak{M}}(X, \mathcal{E})$ is defined as a coarse group $\left(F_{\mathfrak{M}}(X), \mathcal{E}^{\prime}\right)$ such that $(X, \mathcal{E})$ is a subballean of $\left(F_{\mathfrak{M}}(X), \mathcal{E}^{\prime}\right)$ and every coarse mapping $(X, \mathcal{E}) \rightarrow\left(G, \mathcal{E}^{\prime \prime}\right), G \in \mathfrak{M},\left(G, \mathcal{E}^{\prime \prime}\right)$ is a coarse group, can be extended to a coarse homomorphism $\left(F_{\mathfrak{M}}(X), \mathcal{E}^{\prime}\right) \rightarrow\left(G, \mathcal{E}^{\prime \prime}\right)$. The definition implies that a free coarse group is unique up to an asymorphism, which is the identity on $X$.

The following theorem is proved in [21] with explicit description of the coarse structure of $F_{\mathfrak{M}}(X, \mathcal{E})$.

Theorem 12. For every coarse space $(X, \mathcal{E})$ and every non-trivial variety $\mathfrak{M}$ of groups, there exists a free coarse group $F_{\mathfrak{M}}(X, \mathcal{E})$.

2.5. Maximality. A topological space $X$ with no isolated points is called maximal if $X$ has an isolated point in any stronger topology. A topological group $G$ is called maximal if $G$ is maximal as a topological space. Every maximal topological group has an open countable Boolean subgroup, and can be constructed using the Martin Axiom. On the other hand, the existence of a maximal topological group implies the existence of a $P$ point in $\omega^{*}$, see [1]. 
An unbounded coarse space $(X, \mathcal{E})$ is called maximal if $X$ is bounded in every coarse structure $\mathcal{E}^{\prime}$ such that $\mathcal{E} \subset \mathcal{E}^{\prime}$. A coarse group $G$ is called maximal if $G$ is maximal as a coarse space. If a coarse group $(G, \mathcal{I})$ is maximal then $\left\{g^{2}: g \in G\right\}$ is bounded in $(G, \mathcal{I})$, and a maximal coarse Boolean group is constructed in [27, under $C H$, (see also [26. Chapter 10]), but the following question remains open.

Question 2. Does there exist a maximal coarse group in ZFC?

2.6. Normality. We say that subsets $Y, Z$ of a coarse space $(X, \mathcal{E})$ are asymptotically disjoint if, for every $\varepsilon \in \mathcal{E}$ the intersection $B(Y, \varepsilon) \cap B(Z, \varepsilon)$ is bounded.

A subset $U \subset X$ of a coarse space $(X, \mathcal{E})$ is called an asymptotic neighborhood of a set $A \subset X$ if the sets $A$ and $X \backslash U$ are asymptotically disjoint.

A coarse space $(X, \mathcal{E})$ is called normal if any asymptotically disjoint subsets $Y, Z \subset$ $X$ have disjoint asymptotic neighborhoods $O_{Y}, O_{Z}$.

Theorem $13([13)$. For a coarse space $(X, \mathcal{E})$ the following conditions are equivalent:

(1) $(X, \mathcal{E})$ is normal;

(2) for any disjoint and asymptotically disjoint sets $Y, Z \subset X$ there exists a slowly oscillating function $f:(X, \mathcal{E}) \rightarrow[0,1]$ such that $f(Y) \subset\{0\}$ and $f(Z) \subset\{1\}$.

(3) for each subballean $Y$ of $X$, every bounded slowly oscillating function $f: Y \rightarrow \mathbb{R}$ can be extended to a bounded slowly oscillating function on $X$.

We recall that a real-valued function $f: X \rightarrow \mathbb{R}$ defined on a coarse space $(X, \mathcal{E})$ is slowly oscillating if for any $\varepsilon \in \mathcal{E}$ and real number $\delta>0$ there exists a bounded set $B \subset X$ such that $\operatorname{diam} f(B(x, \varepsilon))<\delta$.

Every metrizable coarse space is normal. More generally, a coarse space is normal if its coarse structure has a linearly ordered base, see [13. A partial conversion of this result for products was recently proved in [3].

Theorem 14. If the product $X \times Y$ of two unbounded coarse spaces $X, Y$ is normal, then the coarse space $X \times Y$ has bounded growth and its bornology has a linearly ordered base.

A coarse space $(X, \mathcal{E})$ is defined to have bounded growth if there exists a (multivalued) function $\Phi: X \rightarrow \mathcal{P}_{X}$ such that for every bounded set $B \subset X$ the union $\bigcup_{x \in B} \Phi(x)$ is bounded and for every entourage $\varepsilon \in \mathcal{E}$ there exists a bounded set $D \subset X$ such that $B(x, \varepsilon) \subset \Phi(x)$ for all $x \in X \backslash D$.

Theorem $15([3])$. Let $\kappa$ be an infinite cardinal and $G$ be a group of cardinality $|G| \geq \kappa$, endowed with the coarse structure $\mathcal{E}_{[G]<\kappa}$, generated by the group ideal $[G]<\kappa$. If the coarse space $\left(X, \mathcal{E}_{[G]^{\kappa}}\right)$ is normal (and $G$ is solvable), then $|G|=\kappa$ (and the cardinal $\kappa=|G|$ is regular).

2.7. Coarse structures on topological groups. A subset $A$ of a topological group $G$ (all topological groups are supposed to be Hausdorff) is called totally bounded if, for every neighborhood $U$ of the identity, there exists a finite set $F \subset G$ such that $A \subseteq F U \cap U F$. The group bornology $\mathcal{B}_{\tau}$ of all totally bounded subsets of $(G, \tau)$ defines two (antitall) coarse structures $\mathcal{E}_{l}$ and $\mathcal{E}_{r}$ generated by the ball structures

$\left\{\{(x, y) \in G \times G: y \in\{x\} \cup B x\}: B \in \mathcal{B}_{\tau}\right\}$ and $\left\{\left\{(x, y) \in G \times G: y \in\{x\} \cup B x: B \in \mathcal{B}_{\tau}\right\}\right.$, 
respectively.

The following questions are from [7].

Question 3. Given a group bornology $\mathcal{B}$ on a group $G$, how can one detect whether there exists a group topology $\tau$ on $G$ such that $\mathcal{B}=\mathcal{B}_{\tau}$ ?

Let $(G, \tau)$ be a topological group. We denote by $\tau^{\sharp}$ the strongest group topology on $G$ such that $\mathcal{B}_{\tau^{\sharp}}=\mathcal{B}_{\tau}$, and say that $(G, \tau)$ is b-determined if $\tau^{\sharp}=\tau$. Clearly, every discrete group is $b$-determined. A totally bounded group $G$ is $b$-determined if and only if $\tau$ is the maximal totally bounded topology on $G$.

Question 4. Given a topological group $G$, how can one detect whether $G$ is $b$-determined?

For the coarse structures $\mathcal{E}_{l}, \mathcal{E}_{r}$ and slowly oscillating functions on locally compact groups, see [5].

\section{UNIFORM GROUPS}

We recall that a family $\mathcal{U}$ of subsets of $X \times X$ is a uniformity on a set $X$ if

- $\triangle_{X} \subseteq u$ for each $u \in \mathcal{U}$;

- if $u, v \in \mathcal{U}$ then $u \cap v \in \mathcal{U}$;

- if $u \in \mathcal{U}$ and $u \subseteq v \subset X \times X$ then $v \in \mathcal{U}$.

- for every $u \in \mathcal{U}$, there exists $v \in \mathcal{U}$ such that $v \circ v^{-1} \subseteq u$.

A family $\mathcal{F} \subseteq \mathcal{U}$ is called a base of $\mathcal{U}$ if for every $u \in \mathcal{U}$ there exists $v \in \mathfrak{F}$ such that $v \subseteq u$. A set $X$, endowed with a uniformity $\mathcal{U}$, is called a uniform space.

We say that a uniformity $\mathcal{U}$ on a group $G$ is left (right) invariant if $\mathcal{U}$ has a base consisting of left (right) translation invariant entourages (cf. 2.2).

A filter $\varphi$ on a group $G$ is called a group filter if for every $A \in \varphi$ there exists $B \in \varphi$ such that $B B^{-1} \in \varphi$. In this case every set $A \in \varphi$ contains the unit $e$ of the group $G$. If $\{\varepsilon\} \in \varphi$, then the group filter $\varphi$ is called principal.

Every group filter $\varphi$ determines two uniformities $\mathcal{L}_{\varphi}$ and $\mathcal{R}_{\varphi}$ on $G$ with the bases

$$
\{\{(x, y) \in G \times G: y \in x A\}: A \in \varphi\} \quad \text { and } \quad\{\{(x, y) \in G \times G: y \in A x\}: A \in \varphi\} .
$$

Proposition 3. A uniformity $\mathcal{U}$ on a group $G$ is right invariant if and only if $\mathcal{U}=\mathcal{R}_{\varphi}$ for some group filter $\varphi$ on $G$.

We recall that a topological group $G$ is balanced (= SIN) if the left and right uniformities on $G$ coincide ( $=G$ has a base of invariant neighborhoods of the identity).

Proposition 4. A uniformity $\mathcal{U}$ on a group $G$ is left and right invariant if and only if $G$ endowed with the topology generated by the uniformity $\mathcal{U}$ is a balanced topological group.

Example 2. Let $H$ be a topological group and let $f$ be an arbitrary automorphism of $H$. Then the semidirect product $G=H \lambda\langle f\rangle$ is a right uniform group determined by the filter of neighbourhoods of $e$ in $H$. If $f$ is discontinuous then $G$ is not left uniform. Now let $H$ be the Cartesian product of infinitely many copies of $\mathbb{Z}_{p}$. It is easy to find a discontinuous automorphism of order 2 of $H$. Hence, the right uniform group $G$ contains a compact topological group of index 2 but $G$ is not a topological group. 
We say that a group $G$ is uniformizable if there is a non-principal group filter $\varphi$ on $G$ such that $\cap \varphi=\{e\}$.

We recall that a group $G$ is topologizable if $G$ admits a non-discrete (Hausdorff) group topology. Clearly, every topologizable group is uniformizable, but the class of uniformizable groups is much wider than the class of topologizable groups. By [30, every group can be embedded into some non-topologizable group, and if a group $G$ contains a uniformizable subgroup then $G$ is uniformizable.

Does there exists a non-uniformizable group? In [9], Alexander Olshansky used the following example to construct a countable non-topologizable group.

Example 3. Let $n \geq 2$ be a natural number and $m \geq 665$ be an odd number. Let $A(n, m)$ be the Adian group, see [1]. This group is generated by $n$ elements and has the following properties:

(a) $A(n, m)$ is torsion free;

(b) the center $C$ of $A(n, m)$ is an infinite cyclic group, $C=\langle c\rangle$;

(c) $A(n, m) / C$ is an infinite group of period $m$.

We put $G=A(n, m) /\left\langle c^{m}\right\rangle$, denote by $f: A(n, m) \rightarrow G$ the quotient map and observe that if $g \in G \backslash\{e\}$ then $g$ or $g^{m}$ belongs to the set $\left\{f(c), f\left(c^{2}\right), \ldots, f\left(c^{m-1}\right)\right\}$. It follows that if $\varphi$ is a group filter on $G$ and $\cap \varphi=\{e\}$ then $\{e\} \in \varphi$, so $G$ is non-uniformizable.

Question 5. Does every uniformizable group contain a topologizable subgroup?

Question 6. Can one find a criterion of uniformizability of countable groups in spirit of Markov's criterion of topologizability?

\section{REFERENCES}

1. S. Adian, Classification of periodic words and their applications in group theory, In: Burnside Groups, Proc. Bielefeld, Germany 1977 Workshop, J.L. Mennicke (Ed.), Lecture Notes Math. 806 (1980), 1-40. DOI: 10.1007/BFb0091266

2. T. Banakh, M. Cencelj, D. Repovš, and I. Zarichnyi, Coarse classification of Abelian groups and amenable shift-homogeneous metric spaces, Quaterly. J. Math. 65 (2004), no. 1, $1127-$ 1144. DOI: $10.1093 /$ qmath/hau006

3. T. Banakh and I. V. Protasov, The normality and bounded growth of balleans, Preprint (arXiv:1810.07979).

4. M. Filali, Ie. Lutsenko, and I. Protasov, Boolean group ideal and the ideal structure of $\beta G$, Mat. Stud. 31 (2009), no. 1, 19-28.

5. M. Filali and I. Protasov, Slowly oscillating functions on locally compact groups, Appl. Gen. Topology 6 (2005), no. 1, 67-77.

6. P. Harpe, Topics in geometric group theory, University Chicago Press, 2000.

7. S. Hernandes and I. V. Protasov, Balleans of topological groups, Укр. мат. вісник 8 (2011), no. 1, 87-100; reprinted version: J. Math. Sci. 178 (2011), no. 1, 65-74. DOI: $10.1007 / \mathrm{s} 10958-011-0526-0$

8. N. Hindman and D. Strauss, Algebra in the Stone-Čech compactification, de Gruyter, Berlin, New York, 1998.

9. A. Olshanskii A remark on countable non-topologizable groups, Vestnik Mosk. Gos. Univ. Mat. Mekh. (1980), no. 3, 103 (Russian). 
10. O. V. Petrenko and I. V. Protasov, Balleans and G-spaces, Укр. мат. журн. 64 (2012), nо. 3, 344-350; reprinted version: Ukr. Math. J. 64 (2012), no. 3, 387-393.

DOI: $10.1007 / \mathrm{s} 11253-012-0653-\mathrm{x}$

11. И. В. Протасов, Максималънъе топологии на группах, Сиб. матем. журн. 39 (1998), no. 6, 1368-1381; English version: I. V. Protasov, Maximal topologies on groups, Siberian Math. J. 39 (1998), no. 6, 1184-1194. DOI: 10.1007/BF02674129

12. I. V. Protasov, Morphisms of ball structures of groups and graphs, Укр. мат. журн. 54 (2002), no. 6, 847-855; reprinted version: Ukr. Math. J. 54 (2002), no. 6, 1027-1037. DOI: $10.1023 / \mathrm{A}: 1021772505988$

13. I. V. Protasov, Normal ball structures, Mat. Stud. 20 (2003), no. 1, 3-16.

14. I. V. Protasov, Counting $\Omega$-ideals, Algebra Univers. 62 (2009), no. 4, 339-343. DOI: $10.1007 / \mathrm{s} 00012-010-0032-0$

15. I. V. Protasov, A note on bornologies, Mat. Stud. 49 (2018), no. 1, 13-18. DOI: $10.15330 / \mathrm{ms} .49 .1 .13-18$

16. I. Protasov, Varieties of coarse spaces, Axioms. 7 (2018), no. 2, 32. DOI: $10.3390 /$ axioms7020032

17. I. V. Protasov and T. Banakh, Ball Structures and Colorings of Groups and Graphs, Math. Stud. Monogr. Ser., 11, VNTL, Lviv, 2003.

18. I. Protasov and K. Protasova, Ideals in $P_{G}$ and $\beta G$, Topology Appl. 238 (2018), 24-31. DOI: $10.1016 /$ j.topol.2018.02.003

19. I. V. Protasov and K. D. Protasova, Lattices of coarse structures, Math. Stud. 48 (2017), no. $2,115-123$. DOI: $10.15330 / \mathrm{ms} .48 .2 .115-123$

20. I. Protasov and K. Protasova, Counting coarse subsets of a countable group, Appl. Gen. Topol. 19 (2018), no. 1, 85-90. DOI: 10.4995/agt.2018.7721

21. I. Protasov and K. Protasova, Free coarse groups, arXiv:1803.10504, 2018, preprint.

22. I. V. Protasov and O. I. Protasova, Sketch of group balleans, Math. Stud. 22 (2004), no. 1, 10-20.

23. I. V. Protasov and O. I. Protasova, On closed ideals in $\beta G$, Semigroup Forum 75 (2007), no. 1, 237-240. DOI: $10.1007 / \mathrm{s} 00233-006-0650-1$

24. I. Protasov and S. Slobodianiuk, On asymorphisms of groups, J. Group Theory. 20 (2017), no. 2, 393-399. DOI: 10.1515/jgth-2016-0038

25. I. V. Protasov and A. Tsvietkova, Decomposition of cellular balleans, Topology Proc. 36 (2010) $77-83$.

26. I. Protasov and M. Zarichnyi, General Asymptology, Math. Stud. Monogr. Ser., 12, VNTL, Lviv, 2007.

27. O. V. Protasova, Maximal balleans, Appl. Gen. Topol. 7 (2006), no. 2, 151-163. DOI: 10.4995 /agt.2006.1920

28. J. Roe, Lectures on coarse geometry, AMS University Lecture Ser. 31, Providence, R.I., 2003.

29. S. Shelah, On a problem of Kurosh, Jónsson groups, and applications, Word problems, II (Conf. on Decision Problems in Algebra, Oxford, 1976), pp. 373-394, Stud. Logic Foundations Math., 95, North-Holland, Amsterdam-New York, 1980.

30. A. V. Trofimov, A theorem on embedding into nontopologizable groups, Vestn. Mosk. Univ., Ser. I (2005), no. 3, 60-62 (Russian); English version: Mosc. Univ. Math. Bull. 60 (2005), No. $3,42-44$. 


\section{БОРНОЛОГІЧНІ, ГРУБІ ТА РІВНОМІРНІ СТРУКТУРИ НА ГРУПАХ}

\section{Ігор ПРОТАСОВ}

Киӥвсъкий націоналъний університет імені Тараса Шевченка, Проспект академіка Глушкова, 4d, 03680, Київ, Украӥна e-mail: i.v.protasov@gmail.com

Дано огляд та аналіз різних способів, якими борнології, грубі структури та рівномірності можуть узгоджуватися з груповою операцією.

Ключові слова: борнологія, груба структура, рівномірність, компактифікація Стоуна-Чеха. 